\title{
Joint power and feedback bit allocation for energy- efficient design in limited-feedback coordinated beamforming systems
}

\author{
Fang Yuan ${ }^{*}$ and Chenyang Yang
}

\begin{abstract}
Energy efficiency (EE) has become an important design goal for future cellular systems. In this paper, we study the EE problem for limited-feedback coordinated beamforming systems providing real-time services with constant date rate. To maximize the EE, we jointly optimize the transmit power among users and the feedback bits of each user to quantize the desired and interfering channel directions, subject to the constraints of outage probability and number of feedback bits. An iterative optimization algorithm is proposed for alternating power and bit allocation by employing Yates' framework. To further reduce the computational complexity, a suboptimal algorithm is proposed in closed form by decoupling the problem into subproblems under asymptotical analysis. Simulation results show that the suboptimal algorithm performs closely to the iterative optimization algorithm under high-quantization resolution. Moreover, the proposed two algorithms provide substantial EE gain over existing schemes with equal power and bit allocation, and the EE depends on the target data rate and outage probability of each user as well as the circuit power consumption at the base stations.
\end{abstract}

\section{Introduction}

Explosive growth of high-quality wireless services prefigures that energy saving has become a critical issue $[1,2]$. As a result, it is an urgent task to design energy-efficient communication systems.

Coordinated multi-point transmission with coordinated beamforming (CoMP-CB) can improve spectral efficiency (SE) of cellular networks by avoiding inter-cell interference (ICI), whose performance largely depends on the quality of channel direction information (CDI) available at the base station (BS) [3]. When the CDI cannot be estimated at the BS, e.g., in frequency division duplex systems [4], limited-feedback techniques are often applied, where the CDI is quantized with finite number of bits by each users and fed back to the BS $[5,6]$. Limitedfeedback techniques have been extensively studied for various multi-input multi-output (MIMO) systems [7-9]. In multi-cell systems, equally allocating the bits to quantize the CDIs from multiple BSs is not efficient. Therefore,

*Correspondence: yuanfang@ee.buaa.edu.cn

School of Electronics and Information Engineering, Beihang University, XueYuan Road No.37, 100191, Beijing, China the bit allocation for quantizing multiple CDIs becomes an important issue in multi-cell systems $[5,6,10]$, which differs from the single-cell systems. Various bit allocation schemes were proposed for CoMP-CB systems to minimize the rate loss led by the quantized CDI $[5,6]$ or to maximize the lower bound of per-user rate [10]. These schemes are designed toward maximizing SE, which do not necessarily provide high energy efficiency (EE) [1].

The EE of downlink system is defined as the ratio of system's rate to the power consumption, where the power consumption includes the circuit power and the transmit power [1]. An EE-oriented design needs to consider the feature of the specific traffic in system. As revealed in [11-13], three major capacity measures for system's rate have been defined in the literature to differentiate realtime services from non-real-time services in the physical layer: ergodic capacity, delay-limited capacity $[13,14]$, and outage capacity [15]. Ergodic capacity is suitable for the best-effort services with no delay constraint, and the EE problems for different systems with ergodic capacity were considered in $[1,16]$. The delay-limited capacity characterizes the impact of coding length [15] or the

\section{Springer}

(c) 2015 Yuan and Yang: licensee Springer. This is an Open Access article distributed under the terms of the Creative Commons Attribution License (http://creativecommons.org/licenses/by/4.0), which permits unrestricted use, distribution, and reproduction in any medium, provided the original work is properly credited. 
queueing length [13], and the EE problems for different systems with delay-limited capacity were considered in $[17,18]$. In this paper, we study the energy-efficient limited-feedback CoMP-CB systems with real-time services measured by the outage capacity, where each user has a required quality-of-service $(\mathrm{QoS})$ on the data rate and outage probability. As revealed in [12], the deep fades in wireless channels can be detrimental to real-time services in wireless systems. Compared to the other two capacity measures, the outage capacity is more suitable for constant-rate real-time services under a block-fading channel model [15]. In multi-user limited-feedback systems, each user may need different number of bits for feeding back the CDI, since the feedback channel conditions differ from user to user [19]. These motivate to maximize the EE for limited-feedback CoMP-CB under the constraints on the number of feedback bits for each user in addition to the QoS.

For the considered constant-rate systems, by the definition of EE, maximizing the EE amounts to minimizing the total power consumption at the coordinated BSs [20]. When the circuit power is fixed, minimizing the total power consumption is further equivalent to minimizing the total transmit power. Transmit power minimization problems have been extensively studied in literature under perfect CDI [21]. For example, the works in $[22,23]$ studied the power minimization problems under individual signal-to-interference-and-noise ratio (SINR) constraint. Moreover, the work in [24-26] considered the power minimization problems with outage probability constraints. However, all these works are developed assuming perfect CDI, which do not perform well in practical system with quantized CDI. In limited-feedback systems, the power minimization problems become more challenging, because the impact of imperfect CDI on the system performance should be properly evaluated. The imperfect CDI not only results in many difficulties to analyze the system performances, e.g., outage probability [10,27], but also may make the optimization problems nonlinear even in single-cell systems [28]. In [28], a power allocation algorithm is proposed to minimize the total transmit power for limited-feedback multi-user MIMO systems under individual outage probability constraints. Nevertheless, only the inter-user interference (MUI) is considered in single-cell systems and the impact of ICI is ignored. In addition, the proposed algorithm is given by semi-definite programming (SDP), which is of high complexity [29].

As far as the authors know, no previous work has considered the joint power and feedback bit allocation to improve the system's EE for limited-feedback CoMP-CB under constant date rate services. The EE performance of CoMP-CB is severely limited by two types of residual interferences, i.e., MUI and ICI, which results from the inaccurate coordinated beamforming led by imperfect CDI. The residual interferences can be reduced by allocating more feedback bits for quanting the corresponding CDI or less transmit power to the corresponding interferer. Therefore, allocating the feedback bits and transmit powers in an efficient way eventually improves the system's EE. Motivated by this, we jointly optimize the allocation of transmit powers among multiple users and the feedback bits of each user between quantizing the desired and interfering channels under the constraints on individual feedback overhead and outage probability. To find the optimal solution for the problem, we derive an iterative algorithm using Yates' framework [22]. Since high computational complexity will cause high circuit power consumption, a suboptimal algorithm is developed for the system with high-quantization resolution, where both the power and bit allocation are with closed form expressions of low complexity.

The rest of this paper is organized as follows. We first present the system model in Section 2. In Section 3, we formulate the EE maximization problem and find the optimal solution. Then, in Section 4, a low complexity algorithm is derived. Simulation results are provided in Section 5, and the paper is concluded in the last section.

Notations: ( $)^{T}$ and ()$^{H}$ are respectively the transpose and transpose-conjugate operation. | | denotes the magnitude, \|\| is the two-norm, $[x]^{+}=\max \{0, x\}, \boldsymbol{x} \succ \boldsymbol{y}$ $(\boldsymbol{x} \geq \boldsymbol{y})$ means the vector $\boldsymbol{x}$ is element-wise larger (no less) than the vector $\boldsymbol{y}$, and $\operatorname{Exp}(c)$ means the exponential distribution with mean of $1 / c$.

\section{System model}

\subsection{Signal model}

Consider a cluster of $N_{b}$ BSs each equipped with $N_{t}$ antennas to cooperatively serve $K$ single antenna users in each cell with CoMP-CB [6].

For the $k$ th user served by $\mathrm{BS} b$, user $b_{k}$, the received signal is expressed as

$$
\begin{aligned}
y_{b_{k}}= & \alpha_{b, b_{k}} \boldsymbol{h}_{b, b_{k}}^{H} \sqrt{p_{b_{k}}} \boldsymbol{v}_{b_{k}} s_{b_{k}}+\sum_{m=1, m \neq k}^{K} \alpha_{b, b_{k}} \boldsymbol{h}_{b, b_{k}}^{H} \sqrt{p_{b_{m}}} \boldsymbol{v}_{b_{m}} s_{b_{m}} \\
& +\sum_{c=1, c \neq b}^{N_{b}} \sum_{n=1}^{K} \alpha_{c, b_{k}} \boldsymbol{h}_{c, b_{k}}^{H} \sqrt{p_{c_{n}}} \boldsymbol{v}_{c_{n}} s_{c_{n}}+n_{b_{k}}
\end{aligned}
$$

where $p_{b_{k}}, v_{b_{k}}, s_{b_{k}}$, and $n_{b_{k}}$ are respectively the transmit power, unit-norm precoder, transmit data, and receiver noise, $\alpha_{b, b_{k}}$ is the large-scale fading channel gain between BS $b$ and user $b_{k}$, and $\boldsymbol{h}_{b, b_{k}}$ is the small-scale fading channel vector. Without loss of generalities, we assume $s_{b_{k}}$ and $n_{b_{k}}$ are with unit variance, and $\boldsymbol{h}_{b, b_{k}}$ is subject to independent and identically distributed (i.i.d.) Rayleigh fading. 
The SINR at user $b_{k}$ is

$$
\operatorname{SINR}_{b_{k}}=\frac{p_{b_{k}} \alpha_{b, b_{k}}^{2}\left|\boldsymbol{h}_{b, b_{k}}^{H} \boldsymbol{v}_{b_{k}}\right|^{2}}{1+\sum_{m=1, m \neq k}^{K} I_{b_{m}, b_{k}}+\sum_{c=1, c \neq b}^{N_{b}} \sum_{n=1}^{K} I_{c_{n}, b_{k}}}
$$

where $I_{b_{m}, b_{k}}=p_{b_{m}} \alpha_{b, b_{k}}^{2}\left|\boldsymbol{h}_{b, b_{k}}^{H} \boldsymbol{v}_{b_{m}}\right|^{2}$ is the MUI, and $I_{c_{n}, b_{k}}=$ $p_{c_{n}} \alpha_{c, b_{k}}^{2}\left|\boldsymbol{h}_{c, b_{k}}^{H} \boldsymbol{v}_{c_{n}}\right|^{2}$ is the ICI. We consider $K>1$ and $N_{b}>$ 1 such that both MUI and ICI exist.

In the following, we call $\boldsymbol{h}_{b, b_{k}}$ and $\boldsymbol{h}_{c, b_{k}}$ respectively as the desired and interfering channels for user $b_{k}(c \neq b)$.

\subsection{Limited-feedback transmission model}

We assume that the users have perfect CDI after channel estimation. The CDI is quantized through a given codebook with proper size known by both the BSs and users. Then the codebook indices representing the quantized CDIs of desired and interfering channels of each user are fed back to its serving BS in uplink, after which the CDIs are shared among the BSs for coordinated beamforming.

Take the desired channel $\boldsymbol{h}_{b, b_{k}}$ as an example. Denote the perfect CDI as $\overline{\boldsymbol{h}}_{b, b_{k}}=\overline{\boldsymbol{h}}_{b, b_{k}} /\left\|\boldsymbol{h}_{b, b_{k}}\right\|$ and $\hat{\boldsymbol{h}}_{b, b_{k}}$ as the quantized CDI with unit-norm. The relation between the perfect CDI and quantized CDI can be modeled as [9]

$$
\overline{\boldsymbol{h}}_{b, b_{k}}=\cos \theta_{b, b_{k}} \hat{\boldsymbol{h}}_{b, b_{k}}+\sin \theta_{b, b_{k}} \boldsymbol{q}_{b, b_{k}}
$$

where $\boldsymbol{q}_{b, b_{k}}$ is the error vector of unit-norm, and $\cos \theta_{b, b_{k}}=\left|\overline{\boldsymbol{h}}_{b, b_{k}}^{H} \hat{\boldsymbol{h}}_{b, b_{k}}\right|$ reflects the quantization accuracy.

The average CDI distortion led by quantization is defined as $\mathrm{E}\left\{\sin ^{2} \theta_{b, b_{k}}\right\}=1-\mathrm{E}\left\{\left|\overline{\boldsymbol{h}}_{b, b_{k}}^{H} \hat{\boldsymbol{h}}_{b, b_{k}}\right|^{2}\right\}$ in [8], whose value depends on the employed codebooks, which is intractable for analysis. We therefore consider a quantization cell approximation method used in [9], whose average CDI quantization distortion is

$$
\mathrm{E}\left\{\sin ^{2} \theta_{b, b_{k}}\right\}=\frac{N_{t}-1}{N_{t}} 2^{-\frac{B_{b, b_{k}}}{N_{t}-1}}
$$

where $B_{b, b_{k}}$ is the number of bits for quantizing the CDI of the desired channel $\boldsymbol{h}_{b, b_{k}}$. It was shown in [9] via simulation that Eq. 2 provides an accurate approximation for other codebooks designed for i.i.d. Rayleigh fading channels.

Similarly, we can obtain the average CDI quantization distortion for the interfering channel $\boldsymbol{h}_{c, b_{k}}$ as $\mathrm{E}\left\{\sin ^{2} \theta_{c, b_{k}}\right\}=\frac{N_{t}-1}{N_{t}} 2^{-\frac{B_{c, b_{k}}}{N_{t}-1}}$ for $c \neq b$, where $B_{c, b_{k}}$ is the number of bits for quantizing the CDI of $\boldsymbol{h}_{c, b_{k}}$.

As in most limited-feedback literature $[6,8,19]$, we consider the zero-forcing beamforming (ZFBF) at the BSs which is low complexity but suboptimal and consider full multiplexing, i.e., $N_{t}=N_{b} K$, due to its analytic tractability. Extra transmit antennas at the BSs, i.e., $N_{t}>N_{b} K$, do not necessarily provide higher EE for limited-feedback systems. On one hand, the case $N_{t}>N_{b} K$ introduces extra circuit powers because of extra radio-frequency (RF) chains at the BSs [30]. On the other hand, the quantization distortion in (2) grows exponentially as the number of antennas increases, which can greatly degrade the user SINR [31] and eventually bring down the system EE. Therefore, in this work, we consider only the case of full multiplexing. pause

Denote $\boldsymbol{H}_{b, \ell}=\left[\hat{\boldsymbol{h}}_{b, \ell_{1}}, \cdots, \hat{\boldsymbol{h}}_{b, \ell_{K}}\right]$ as the channel matrix from BS $b$ to the users served by BS $\ell(b, \ell=$ $\left.1, \ldots, N_{b}\right)$. Then the ZFBF precoder at BS $b$ for user $b_{k}$ in the CoMP-CB system, $\boldsymbol{v}_{b_{k}}$, is obtained from normalizing the $k$ th column of $\left(\mathcal{H}_{b}^{H} \mathcal{H}_{b}\right)^{-1} \mathcal{H}_{b}$ where $\mathcal{H}_{b}=$ $\left[\boldsymbol{H}_{b, b}, \boldsymbol{H}_{b, 1}, \cdots, \boldsymbol{H}_{b, b-1}, \boldsymbol{H}_{b, b+1} \cdots, \boldsymbol{H}_{b, N_{b}}\right]$.

\section{EE optimization for limited-feedback CoMP-CB system}

In this section, we study the bit and power allocation problem to maximize the downlink EE of the CoMP-CB system, where the total number of feedback bits of each user is given. We consider the system providing real-time services under a block fading channel, where each user requires a constant data rate. Since the data rate requirement of each user may not be supported under fading channel with imperfect CDI, to guarantee the QoS, a constraint on outage probability is considered, which is assumed less than a target value.

\subsection{Problem formulation}

Denote $\gamma_{b_{k}}$ as the targeted SINR of user $b_{k}$ for the constant data rate in downlink. Then, the outage probability of user $b_{k}$ is defined as

$$
\mathrm{P}_{\text {out }}\left(\gamma_{b_{k}}\right)=\operatorname{Pr}\left\{\operatorname{SINR}_{b_{k}}<\gamma_{b_{k}}\right\} .
$$

Considering the outage probability is required less than a target value $O_{b_{k}}$, i.e., $\mathrm{P}_{\text {out }}\left(\gamma_{b_{k}}\right) \leq O_{b_{k}}$, the required data rate by user $b_{k}$, i.e., outage capacity, is given as

$$
R_{b_{k}}=\log \left(1+\gamma_{b_{k}}\right)\left(1-O_{b_{k}}\right) .
$$

The EE of downlink CoMP-CB system is defined as the ratio of the system's sum rate to the total power consumption in downlink [32,33], which is,

$$
E E=\frac{\sum_{b=1}^{N_{b}} \sum_{k=1}^{K} R_{b_{k}}}{N_{b} p_{c}+\sum_{b=1}^{N_{b}} \sum_{k=1}^{K} p_{b_{k}}}
$$

where $p_{c}$ is the circuit power at each BS. The modeling of circuit power can be found in [34], which includes the power consumed by RF chain, signal processing, cooling, etc. at each BS as well as that by the backhaul links for channel sharing.

With zero-forcing beamforming (ZFBF), it was shown in [35] that the interference powers $\left|\boldsymbol{h}_{b, b_{k}}^{H} \boldsymbol{v}_{b_{m}}\right|^{2}$ and $\left|\boldsymbol{h}_{c, b_{k}}^{H} \boldsymbol{v}_{c_{n}}\right|^{2}$ are exponentially distributed respectively with 
probability density distribution (PDF) of $\operatorname{Exp}\left(1 / \delta_{b, b_{k}}\right)$ and $\operatorname{Exp}\left(1 / \delta_{c, b_{k}}\right)$, where their expectations are

$$
\delta_{b, b_{k}}=2^{\frac{-B_{b, b_{k}}}{N_{t}-1}} \text { and } \delta_{c, b_{k}}=2^{\frac{-B_{c, b_{k}}}{N_{t}-1}} .
$$

Moreover, when $N_{b} K=N_{t}$, the precoder $\boldsymbol{v}_{b_{k}}$ is independent with $\boldsymbol{h}_{b, b_{k}}$ and the variable $\left|\boldsymbol{h}_{b, b_{k}}^{H} \boldsymbol{v}_{b_{k}}\right|^{2}$ is exponentially distributed with PDF of $\operatorname{Exp}(1)$ [9]. Then, the outage probability of user $b_{k}$ under given transmit powers is [10]

$$
\frac{\mathrm{P}_{\text {out }}\left(\gamma_{b_{k}}\right)=1-}{e^{-\frac{\gamma_{b_{k}}}{\alpha_{b, b_{k}}^{2} p_{b_{k}}}}} \frac{\prod_{m=1, m \neq k}^{K}\left(1+\frac{\gamma_{b_{k}} \delta_{b, b_{k}} p_{b_{m}}}{p_{b_{k}}}\right) \prod_{c=1, c \neq b}^{N_{b}} \prod_{n=1}^{K}\left(1+\frac{\gamma_{b_{k}} \delta_{c, b_{k}} \alpha_{c, b_{k}}^{2} p_{c_{n}}}{\alpha_{b, b_{k}}^{2} p_{b_{k}}}\right)}{N^{\prime}}
$$

which depends on the transmit power, average CDI quantization distortion and target SINR of the user.

By observing (5), we can find that limited feedback has a large impact on the outage probability. The impact of MUI and ICI is conveyed by the denominator of (5). Given the transmit powers, the larger average CDI distortion $\delta_{b, b_{k}}$ or $\delta_{c, b_{k}}$ is, the larger MUI or ICI the user $b_{k}$ experiences, and the larger the outage probability becomes. In contrast, under perfect CDI, the MUI and ICI become zero and the denominator of (5) is unit, which means the bit allocation is not necessary.

To maximize the EE of downlink CoMP-CB, the problem of jointly allocating the transmit powers among multiple users and the total number of bits of each user between quantizing its desired and interfering CDIs can be formulated as

$$
\begin{array}{rl}
\max _{p_{b_{k}}, \boldsymbol{B}_{b_{k}}} & E E \\
\text { s.t. } & \mathrm{P}_{\text {out }}\left(\gamma_{b_{k}}\right) \leq O_{b_{k}}, \quad \forall b_{k} \\
& \mathbf{1}^{T} \boldsymbol{B}_{b_{k}} \leq B_{b_{k}}, \quad \forall b_{k} \\
& p_{b_{k}}>0, \quad \forall b_{k} \\
& \boldsymbol{B}_{b_{k}} \geq \mathbf{0}, \quad \forall b_{k}
\end{array}
$$

where $\boldsymbol{B}_{b_{k}} \triangleq\left[B_{1, b_{k}}, B_{2, b_{k}}, \cdots, B_{N_{b}, b_{k}}\right]^{T}$ is the bit allocation profile of user $b_{k}$ for quantizing the CDIs from desired and interfering BSs, $B_{b_{k}}$ is the total number of feedback bits of user $b_{k}, \mathbf{1}$ and $\mathbf{0}$ are respectively the all-one and all-zero vectors. The QoS requirement parameters $\gamma_{b_{k}}$ and $O_{b_{k}}$ are constant and pre-specified by the system. In the constraint (6b), as shown in (4), allocating more bits for $B_{b, b_{k}}$ in $\boldsymbol{B}_{b_{k}}$ can lower down the average MUI, while allocating more bits for $B_{c, b_{k}}(c \neq b)$ in $\boldsymbol{B}_{b_{k}}$ can lower down the $c$ th average ICI. Therefore, the bit allocation in (6) is to improve the system EE by considering both the impact of MUI and ICI.
The optimization problem in (6) does not associate with the instantaneous channel information. As a result, the power and bit allocation does not need a frequent design, which will not causing too much signaling overhead. Nonetheless, if we consider the maximum transmit power constraint at each $\mathrm{BS}$, the problem will become infeasible when the target date rate is high, even with perfect CDI. To demonstrate the EE potential of the CoMP-CB system and differentiate the infeasibility led by the transmit power and imperfect CDI, we do not take into account the maximum transmit power constraint.

\subsection{Optimal solution of joint power and bit allocation}

Since each user's date rate $R_{b_{k}}$ is constant in the numerator of EE and the circuit power does not depend on the variables to be optimized, problem (6) is equivalent to the following joint power and bit allocation problem that minimizes the total transmit power

$$
\begin{aligned}
\min _{p_{b_{k}}, \boldsymbol{B}_{b_{k}}} & \sum_{b=1}^{N_{b}} \sum_{k=1}^{K} p_{b_{k}} \\
\text { s.t. } & (6 \mathrm{a}),(6 \mathrm{~b}),(6 \mathrm{c}),(6 \mathrm{~d}) .
\end{aligned}
$$

It should be noted that although the equivalence between the problems (6) and (7) is applied here to reduce the complexity of optimization problem, the work in this paper is motivated by improving the system EE as defined in (3). Moreover, to show the impact of proposed algorithms for joint power and bit allocation, and the other impacts such as circuit power and outage probability, the system EE in (3) rather than total transmit power in (7) will be mainly evaluated in the simulation section.

The optimization problem in (7) is nonlinear. To see this, from (5), we can rewrite the constraint (6a) as

$$
\begin{aligned}
& p_{b_{k}} \geq\left(\frac{\gamma_{b_{k}}}{\alpha_{b, b_{k}}^{2}}+\sum_{m=1, m \neq k}^{K} p_{b_{k}} \log \left(1+\frac{\gamma_{b_{k}} \delta_{b, b_{k}} p_{b_{m}}}{p_{b_{k}}}\right)\right. \\
& \left.+\sum_{c=1, c \neq b}^{N_{b}} \sum_{n=1}^{K} p_{b_{k}} \log \left(1+\frac{\gamma_{b_{k}} \delta_{c, b_{k}} \alpha_{c, b_{k}}^{2} p_{c_{n}}}{\alpha_{b, b_{k}}^{2} p_{b_{k}}}\right)\right) \frac{1}{\log \frac{1}{1-O_{b_{k}}}} \\
& \triangleq \boldsymbol{T}_{b_{k}}\left(\boldsymbol{p}, \boldsymbol{B}_{b_{k}}\right)
\end{aligned}
$$

where $\boldsymbol{p} \triangleq\left[p_{1_{1}}, \cdots, p_{1_{K}}, \cdots, p_{N_{b 1}}, \cdots, p_{N_{b K}}\right]^{T}$ denotes the power profile for all $K N_{b}$ users. All the variables in (8), including the transmit powers and the bits conveyed in average CDI distortions, are nonlinear in the constraint.

Solving the problem (7) is quite challenging due to its nonlinearity. Instead of solving it directly, here, we study an equivalent problem for (7) and reveal that the nonlinear optimization problem can be solved by using a standard iteration algorithm. 
The nonlinear optimization problem (7) is equivalent to the following problem

$$
\begin{aligned}
\min _{p_{b_{k}}} & \sum_{b=1}^{N_{b}} \sum_{k=1}^{K} p_{b_{k}} \\
\text { s.t. } & p_{b_{k}} \geq \boldsymbol{T}_{b_{k}}(\boldsymbol{p}), \quad \forall b_{k} \\
& p_{b_{k}}>0, \quad \forall b_{k}
\end{aligned}
$$

where the function $\boldsymbol{T}_{b_{k}}(\boldsymbol{p})$ is defined through the optimization of bit allocation

$$
\boldsymbol{T}_{b_{k}}(\boldsymbol{p}) \triangleq \min _{\mathbf{1}^{T} \boldsymbol{B}_{b_{k}} \leq B_{b_{k}}, \boldsymbol{B}_{b_{k}} \succeq 0} \boldsymbol{T}_{b_{k}}\left(\boldsymbol{p}, \boldsymbol{B}_{b_{k}}\right) .
$$

The equivalence comes from the fact that the bit allocation profile $\boldsymbol{B}_{b_{k}}$ of user $b_{k}$ only affects $\boldsymbol{T}_{b_{k}}\left(\boldsymbol{p}, \boldsymbol{B}_{b_{k}}\right)$. Considering the inequality (8), the minimum value of $p_{b_{k}}$ is the one satisfying the constraint in (10a) among all the possible values of $\boldsymbol{T}_{b_{k}}\left(\boldsymbol{p}, \boldsymbol{B}_{b_{k}}\right)$ with different bit allocation profile $\boldsymbol{B}_{b_{k}}$ for user $b_{k}$. Therefore, the two problems are equivalent in the sense that they have the same globally optimal solution.

The optimal solution to problem (10) can be found by alternatively optimizing the bit and power allocation.

\subsubsection{Bit allocation with given power allocation}

In each iteration with given transmit powers, the bit allocation for quantizing the desired and interfering CDIs of user $b_{k}$ can be found from the following problem (i.e., (11)),

$$
\begin{aligned}
\min _{\boldsymbol{B}_{b_{k}}} & \boldsymbol{T}_{b_{k}}\left(\boldsymbol{p}, \boldsymbol{B}_{b_{k}}\right) \\
\text { s.t. } & \boldsymbol{1}^{T} \boldsymbol{B}_{b_{k}} \leq B_{b_{k}}, \boldsymbol{B}_{b_{k}} \succeq 0
\end{aligned}
$$

It is easy to verify that this problem is convex on the $\boldsymbol{B}_{b_{k}}$. Therefore, the optimal solution can be obtained by using standard convex optimization tools [29].

\subsubsection{Power allocation with given bit allocation}

When the bit allocation for each user is obtained, the power allocation for multiple users can be optimized by using Yates' framework [22], which is a fixed-point iteration problem. To show the convergence of the proposed iterative algorithm, we provide the following proposition.

Proposition 1. $T_{b_{k}}(\boldsymbol{p})$ is a standard interference function of the vector $\boldsymbol{p}$, i.e., it satisfies the following three properties:

1. Positivity: $\boldsymbol{T}_{b_{k}}(\boldsymbol{p})>0$.

2. Monotonicity: $\boldsymbol{T}_{b_{k}}(\boldsymbol{p})$ is monotonic with each element in $\boldsymbol{p}$.

3. Scalability: $\boldsymbol{T}_{b_{k}}(\tau \boldsymbol{p})<\tau \boldsymbol{T}_{b_{k}}(\boldsymbol{p})$ for any constant $\tau>1$.

The proposition can be proved using a similar method as that in [25].
Yates found that for any function used for iteration, if the function satisfies all the properties of a standard interference function [22], the iteration will converge to the globally optimal solution. Using Yates' framework, we can update the transmit powers with the solution of bit allocation obtained from problem (12) as

$$
p_{b_{k}}=\boldsymbol{T}_{b_{k}}(\boldsymbol{p})
$$

The iterative algorithm to solve problem (10) is summarized in Algorithm 1, which converges to optimal solution and provides a performance baseline. However, the iteration in the algorithm increases the computational complexity for signal processing and thus may increase the circuit power consumption. Therefore, we further propose a non-iterative algorithm with lower complexity in the sequel.

\section{Algorithm 1 Iterative algorithm for finding optimal solution}

Initialize: $i=0, p_{b_{k}}^{0} B_{b_{k}}^{0} \succeq 0$

Iterate for each user $b_{k}$

1. update $i=i+1$;

2. update bit allocation $\boldsymbol{B}_{b_{k}}^{i}$ for user $b_{k}$ by solving problem (12) with given $p_{b_{k}}^{i-1}$;

3. update power allocation $\boldsymbol{p}_{b_{k}}^{i}$ by (13) with given $\boldsymbol{B}_{b_{k}}^{i}$ and $\boldsymbol{p}_{b_{k}}^{i-1}$;

Terminate until convergence or reaching maximum number of iterations.

\section{Low complexity algorithm}

In this section, we propose a suboptimal but low complexity algorithm to solve problem (10). By using asymptotical analysis and introducing upper bound, we decouple the joint bit and power allocation problem into two subproblems: 1) optimizing power allocation with given bit allocation, and 2) optimizing bit allocation with optimized power allocation. The two subproblems are solved without the need for iteration.

\subsection{Power allocation with given bit allocation}

We first optimize the power allocation with given bit allocation for each user.

Using the inequality (14) in [36], we can obtain a upper bound for outage probability in (5) as

$$
\mathrm{P}_{\text {out }}\left(\gamma_{b_{k}}\right) \leq 1-e^{-\frac{\gamma_{b_{k}}}{p_{b_{k}}} \boldsymbol{T}_{b_{k}}^{r}\left(\boldsymbol{p}, \boldsymbol{B}_{b_{k}}\right)}
$$

where the function $T_{b_{k}}^{r}\left(\boldsymbol{p}, \boldsymbol{B}_{b_{k}}\right)$ is written as

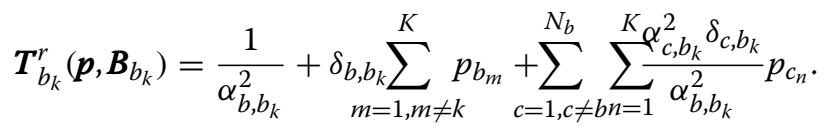


With this upper bound, we formulate a power allocation problem with given bit allocation as

$$
\begin{array}{ll}
\min _{p_{b_{k}}} & \sum_{b=1}^{N_{b}} \sum_{k=1}^{K} p_{b_{k}} \\
\text { s.t. } & 1-e^{-\frac{\gamma_{b_{k}}}{p_{b_{k}}} \boldsymbol{T}_{b_{k}}^{r}\left(\boldsymbol{p}, \boldsymbol{B}_{b_{k}}\right)} \leq O_{b_{k}}, \quad \forall b_{k} \\
& p_{b_{k}}>0, \quad \forall b_{k}
\end{array}
$$

whose solution is suboptimal for the original problem (10). Specially, we can rewrite the constraint (16a) as

$$
p_{b_{k}} \geq \gamma_{b_{k}}^{r} \boldsymbol{T}_{b_{k}}^{r}\left(\boldsymbol{p}, \boldsymbol{B}_{b_{k}}\right)
$$

where $\gamma_{b_{k}}^{r} \triangleq \frac{-\gamma_{b_{k}}}{\log \left(1-O_{b_{k}}\right)}$

Now both the objective function and constraints in (16) are expressed as linear functions of the optimization variables, i.e., the elements in $\boldsymbol{p}$. Define a constant matrix $\boldsymbol{A}$ of size $K N_{b} \times K N_{b}$ with the $(i, j)$ th element as

$$
\boldsymbol{A}_{i, j}=\left\{\begin{array}{cl}
0, & \text { if } j=i \\
\gamma_{b_{k}}^{r} \delta_{b, b_{k}}, & \text { if } K(b-1)<j \leq K b, j \neq i \\
\frac{\gamma_{b_{k}}^{r} \alpha_{c, b_{k}}^{2} \delta_{c, b_{k}}}{\alpha_{b, b_{k}}^{2}}, & \text { if } j \leq K(b-1), \text { or } j>K b
\end{array}\right.
$$

and a constant vector $\boldsymbol{w}$ of size $K N_{b} \times 1$ with the $i$ th element as

$$
\boldsymbol{w}_{i}=\frac{\gamma_{b_{k}}^{r}}{\alpha_{b, b_{k}}^{2}}
$$

where $c \neq b$ and the index $i=(b-1) K+k, b=1, \cdots, N_{b}$, $k=1, \cdots, K$. The matrix $\boldsymbol{A}$ describes the coefficients of the variables (e.g., transmit powers) in the constraint (17). The vector $\boldsymbol{w}$ describes the constants irrespective of variables in the constraint (17). Therefore, with the upper bound in (14), problem (16) becomes a linear programming problem for the transmit powers, which has a much lower complexity than the problem (7).

With the definition of $\boldsymbol{A}$ and $\boldsymbol{w}$, we can immediately write the problem (16) into a standard linear programming problem, which is

$$
\begin{aligned}
\min _{\boldsymbol{p}} & \mathbf{1}^{T} \boldsymbol{p} \\
\text { s.t. } & \boldsymbol{p} \succeq \boldsymbol{A} \boldsymbol{p}+\boldsymbol{w}, \text { and } \boldsymbol{p} \succ \mathbf{0} .
\end{aligned}
$$

From [29], the optimal solution of problem (20) can be obtained with closed form as

$$
\boldsymbol{p}=(\boldsymbol{I}-\boldsymbol{A})^{-1} \boldsymbol{w}
$$

and the corresponding minimum sum transmit power of all the BSs becomes

$$
p_{S}=\mathbf{1}^{T}(\boldsymbol{I}-\boldsymbol{A})^{-1} \boldsymbol{w} .
$$

There are two benefits of the solution in (21). One is that linear programming is desirable for low complexity in computation. The other is that the solution is of explicit form, which is desirable for the performance analysis in the following.

\subsection{Bit allocation with optimized power allocation}

We then optimize the bit allocation between the desired and interfering CDIs to further reduce the minimum total transmit power $p_{S}$. Unfortunately, the optimal bit allocation that minimizes $p_{S}$ is hard to find because the expression of $p_{S}$ in (22) is complicated and nonlinear with the CDI quantization distortion. In the sequel, we study the bit allocation problem resorting to asymptotic analysis.

Proposition 2. The minimum total transmit power in (22) satisfies

$$
\lim _{\delta \rightarrow 0} \frac{p_{S}}{p_{\mathrm{CDI}}+\sum_{b=1}^{N_{b}} \sum_{k=1}^{K} \frac{\gamma_{b_{k}}^{r}}{\alpha_{b, b_{k}}^{2}} \Delta_{b_{k}}}=1
$$

where $\delta=\max _{\ell, b_{k}} \delta_{\ell, b_{k}}, p_{\mathrm{CDI}}=\sum_{b=1}^{N_{b}} p_{s, b}$ with $p_{s, b}=$ $\sum_{m=1}^{K} \frac{\gamma_{b_{m}}^{r}}{\alpha_{b, b_{m}}^{2}}, \Delta_{b_{k}}=\sum_{\ell=1}^{N_{b}} g_{s, \ell} \alpha_{\ell, b_{k}}^{2} \delta_{\ell, b_{k}}$ with

$$
g_{s, \ell}=\left\{\begin{array}{c}
p_{s, b}-\frac{\gamma_{b_{k}}^{r}}{\alpha_{b, b_{k}}^{2}}, \quad \ell=b \\
p_{s, \ell}, \quad \ell \neq b
\end{array}\right.
$$

Proof: When $\delta \rightarrow 0$, it can be verified that $\|\boldsymbol{A}\| \rightarrow 0$, using the Taylor series, we obtain the equivalent infinitesimal of $(\boldsymbol{I}-\boldsymbol{A})^{-1}$ from the first order expansion as [37]

$$
(\boldsymbol{I}-\boldsymbol{A})^{-1} \approx \boldsymbol{I}+\boldsymbol{A} .
$$

Using the equivalent infinitesimal replacement in the limit operation and the definition of $\boldsymbol{A}$ and $\boldsymbol{w}$, we have

$$
\begin{aligned}
& \lim _{\delta \rightarrow 0} \frac{p_{S}}{\mathbf{1}^{T}(\boldsymbol{I}-\boldsymbol{A})^{-1} \boldsymbol{w}}=\lim _{\delta \rightarrow 0} \frac{p_{S}}{p_{S}^{T}(\boldsymbol{I}+\boldsymbol{A}) \boldsymbol{w}} \\
& =\lim _{\delta \rightarrow 0} \frac{p_{\mathrm{CDI}}+\sum_{b=1}^{N_{b}} \sum_{k=1}^{K} \frac{\gamma_{b_{k}}^{r}}{\alpha_{b, b_{k}}^{2}} \Delta_{b_{k}}}{p_{b_{k}}}
\end{aligned}
$$

Now, we complete the proof for the proposition.

The condition $\delta \rightarrow 0$ requires that the system is with high-quantization resolution for each CDI. With perfect CDI, it is easy to show that the minimum total transmit power is $p_{\mathrm{CDI}}=\sum_{b=1}^{N_{b}} p_{s, b}$. When the CDI is imperfect, the transmit power will increase in order to achieve the same target SINR and outage probability under the residual MUI and ICI, which leads to the term $\sum_{b=1}^{N_{b}} \sum_{k=1}^{K} \frac{\gamma_{b_{k}}^{r}}{\alpha_{b, b_{k}}^{2}} \Delta_{b_{k}}$ in the dominator in (25). This fraction of transmit power reduces with the improved CDI quantization accuracy, and thereby can be minimized by the bit allocation.

According to Proposition 2, to improve the EE for the system with high-quantization resolution, we only need to 
minimize $\sum_{b=1}^{N_{b}} \sum_{k=1}^{K} \frac{\gamma_{b_{k}}^{r}}{\alpha_{b, b_{k}}^{2}} \Delta_{b_{k}}$. Considering the individual constraint imposed on the number of bits for each user, and considering that only $\Delta_{b_{k}}$ is related with the bit allocation of user $b_{k}$, the power allocation problem can be formulated as

$$
\begin{aligned}
& \min _{\boldsymbol{B}_{b_{k}}} \Delta_{b_{k}}=\sum_{\ell=1}^{N_{b}} g_{s, \ell} \alpha_{\ell, b_{k}}^{2} \delta_{\ell, b_{k}} \\
& \text { s.t. } \quad \mathbf{1}^{T} \boldsymbol{B}_{b_{k}} \leq B_{b_{k}}, \text { and } \boldsymbol{B}_{b_{k}} \succeq 0 .
\end{aligned}
$$

This is a convex optimization problem. First, the constraints are linear and thus convex. Second, the objective function is a weighted sum of exponential functions with positive weights, which is also convex. The solution can be found from the Karush-Kuhun-Tucker (KKT) conditions, which turns into a water-filling algorithm with the unique solution as follows:

$$
B_{\ell, b_{k}}=\left(N_{t}-1\right)\left[\log _{2} g_{s, \ell}+\log _{2} \alpha_{\ell, b_{k}}^{2}-\log _{2} v\left(N_{t}-1\right)\right]^{+}
$$

where $v>0$ should satisfy $\sum_{\ell=1}^{N_{b}} B_{\ell, b_{k}}=B_{b_{k}}$.

The result in (27) shows that the allocated number of bits for quantizing the CDI from the $\ell$ th BS to user $b_{k}$ not only increases with the large-scale fading gain $\alpha_{\ell, b_{k}}^{2}$ but also grows with the parameter $g_{s, \ell}$ defined in (23), which is associated with the QoS requirements (i.e., $\gamma_{\ell_{k}}$ and $O_{\ell_{k}}$ ) for the users served in the $\ell$ th cell.

To illustrate this, we consider the case that the QoS requirements for users in the same cell are identical, i.e., $\gamma_{\ell_{k}}^{r}=\gamma_{\ell}^{r}$ for all $k$, then (23) reduces to

$$
g_{s, \ell}=\left\{\begin{array}{cc}
\gamma_{b}^{r}\left(\sum_{m=1}^{K} \frac{1}{\alpha_{b, b_{m}}^{2}}-\frac{1}{\alpha_{b, b_{k}}^{2}}\right), & \ell=b \\
\gamma_{\ell}^{r} \sum_{m=1}^{K} \frac{1}{\alpha_{\ell, \ell_{m}}^{2}}, & \ell \neq b
\end{array}\right.
$$

It means that the parameters $g_{s, \ell}$ are linear with its own QoS requirement $\gamma_{\ell}^{r}$ in the $\ell$ th cell. Consequently, we can find that (27) tends to allocate more bits to the cell with larger QoS requirement. By contrast, the bit allocation algorithm for improving SE proposed in $[5,6]$ only exploits the receive powers in the desired and interfering channels but does not consider the different QoS requirements, and thus cannot be applied in this work.

For general setting of QoS requirement $\gamma_{\ell_{k}}^{r}$, the parameter $g_{s, \ell}$ measures the interference condition from the $\ell$ th BS to the user $b_{k}$ quantitatively. Observe $g_{s, \ell}$ defined in (23): when $\ell=b$, it equals to $g_{s, b}=\sum_{m=1, m \neq k}^{K} \frac{\gamma_{b_{m}}^{r}}{\alpha_{b, b_{m}}^{2}}$, which is a sum of $K-1$ items coming from the $K-1$ MUIs experienced by user $b_{k}$ from the $b$ th BS; when $\ell \neq b$, it equals to $g_{s, \ell}=\sum_{m=1}^{K} \frac{\gamma_{\ell_{m}}^{r}}{\alpha_{\ell, \ell_{m}}^{2}}$, which is a sum of $K$ items coming from the ICI of $K$ users served by the $\ell$ th BS.
Therefore, both impacts of MUI and ICI are reflected in the bit allocation (27).

The low complexity algorithm is summarized in Algorithm 2, where the power allocation (21) depends on the bit allocation algorithm (27). It will be shown in the simulation section that under high-quantization resolution, the performance of suboptimal algorithm is close to that of optimal algorithm.

\section{Algorithm 2 Low complexity algorithm}

For each user $b_{k}$,

1. bit allocation: obtain $\boldsymbol{B}_{b_{k}}$ with (27);

2. power allocation with the bit allocation in (27): obtain $p_{b_{k}}$ with (21).

\subsection{Feasibility of the proposed algorithms}

The bit and power allocation algorithms are derived under the implicit assumption that the optimal problems in (10) and (20) are feasible, i.e., the allocated powers are neither negative nor infinity. However, as indicated by [22], in some cases with strong interference, the optimal solutions are not feasible.

A sufficient and necessary condition for the feasibility of problem (20) is that the maximum eigenvalue of $\boldsymbol{A}$ should be less than one [22]. Therefore, the solution in (21) is applicable only when the maximum eigenvalue of $A$ is less than one.

This is also a sufficient condition for the feasibility of problem (10) (equivalently, original problem (6)), since the solution of problem (20) is an upper bound of the solution of problem (10). The exact sufficient and necessary condition for problem (10) is generally unknown. As a result, in some cases the suboptimal power allocation in (21) may not be feasible when the optimal power allocation in (13) is feasible.

\section{Simulation results}

In this section, we evaluate the performance of the proposed algorithms through simulations.

\subsection{Simulation setup}

We consider a CoMP-CB system with a cooperative cluster size of three, i.e., $N_{b}=3$. The inter-cluster interference is modeled as noise, which is reflected in the noise power set as $-104 \mathrm{dBm}$. The cell radius is $250 \mathrm{~m}$, the path loss factor is 3.76 , and the downlink transmission bandwidth is $10 \mathrm{Mhz}$. The small-scaling fading channels are subject to i.i.d. Rayleigh flat fading. Random vector quantization (RVQ) codebooks are applied for quantizing each CDI.

We assume there are two users in each cell, i.e., $K=$ 2 , and the number of antennas at each BS is six, i.e., 
$N_{t}=6$. It means each user experiences one MUI interference from the other user in the same cell and four ICI interferences from the users in two neighboring cells.

The system topology is shown in Figure 1, where two scenarios about user locations are studied. The first scenario is with fixed user location, which is applied in Figures $2,3,4,5,6,7$. In this scenario, two users in each cell are located on the line between their serving BS and the point $O$ and with a distance $d$ to their serving BS and a distance $d_{I}$ to the other two interfering BSs. Moreover, the users in three cells are symmetrically distributed. The major benefit of this scenario is that we only need to employ one single parameter $d$ to reflect user location, which determines the large-scale channel gains of desired and interfering channels. When changing the distance $d$ to the serving BS, the large-scale channel gains of desired and interfering channels are changed simultaneously which are unequal except for the user location $d=250 \mathrm{~m}$. The different impacts of desired and interfering channels are reflected by the heterogeneous large-scale channel gains from desired and interfering BSs, which are exploited in the power and bit allocation by the two proposed algorithms. The second scenario is with random user locations, which is applied in Figure 8 to show the impact of general user locations. In the second scenario, each user is randomly dropped in the CoMP region shown in Figure 1. The CoMP region is defined as the cell-edge region, where the inter-cell interference is strong and coordinated beamforming is necessary.

To reflect different data rate requirements of each user, we set the data rate constraint in the three cells as $R_{1_{1}}=$ $R_{1_{2}}=\rho_{1} R(1-O), R_{2_{1}}=R_{2_{2}}=\rho_{2} R(1-O)$, and $R_{3_{1}}=$ $R_{3_{2}}=\rho_{3} R(1-O) \mathrm{bps} / \mathrm{hz}$, where $\sum_{i=1}^{3} \rho_{i}=1$. Then, the total date rate of all the six users is $2 R(1-O)$. Unless otherwise specified, the target outage probability for each user is $O=0.1$.

Similar simulation results can be observed for more complex cases with arbitrary size of cluster, random located users and arbitrary data rate requirement, which are not shown for conciseness.

For simplicity, in the following, we refer to the optimal power and bit allocation obtained from the iterative algorithm in Algorithm 1 as 'OPA+OBA', and the suboptimal power and bit allocation obtained from the low complexity algorithm in Algorithm 2 as 'SPA+SBA', both in the main text and in the legend. The obtained bit allocation results in the optimization problems are rounded into nearest integers as the number of allocated bits.

We assume that the total number of feedback bits for each user is conveyed via $N_{s}$ slots in uplink. The number of feedback bits in each slot is adaptive to the average uplink signal-to-noise ratio (SNR), which is determined by the constraint $\operatorname{P}_{r}\left\{\log _{2}\left(1+\mathrm{SNR}_{b_{k}}^{u}\right) \leq \frac{B_{b_{k}}}{N_{s}}\right\} \leq 0.1$, where
$\mathrm{SNR}_{b_{k}}^{u}$ is the instantaneous receive SNR at the BS $b$ from user $b_{k}$. We set $N_{s}=3\left(N_{t}-1\right)$ in Subsection 5.2 and the impact of the value of $N_{s}$ on the uplink and downlink energy consumptions will be investigated in Subsection 5.3. It is worthy to note that when the value of $N_{s}$ changes, the total number of feedback bits for each user changes.

Denote uplink transmit power as $p^{u}$, which is set as 26 $\mathrm{dBm}$ (approximately $400 \mathrm{mw}$ ). Let $\boldsymbol{h}_{b, b_{k}}^{u}$ be the instantaneous uplink small-scale fading channel vector. Then, the uplink receiving $\mathrm{SNR}$ is $\mathrm{SNR}_{b_{k}}^{u}=p^{u} \alpha_{b, b_{k}}^{2}\left\|\boldsymbol{h}_{b, b_{k}}^{u}\right\|^{2}$. The large-scale fading gains of the uplink and downlink channels are equal and the small-scaling fading uplink channels are subject to i.i.d. Rayleigh flat fading and independent with the downlink channels.

\subsection{Validation of upper bound and asymptotic analysis}

To validate the upper bound and asymptotic analysis, we compare the total transmit power at all coordinated BSs obtained from the iterative algorithm in Algorithm 1, the low complexity algorithm in Algorithm 2, and the asymptotical analysis in Proposition 2 using the bit allocation in (27).

As shown in Figure 2, the low complexity algorithm performs close to the iterative optimization algorithm as the number of feedback bits for each user increases, which indicates that using the upper bound of outage probability in (14) leads to minor performance loss. Moreover, as the number of bits increases, the performance of both algorithms converge to the limit result of asymptotic analysis in Proposition 2.

\subsection{Performance evaluation of the proposed algorithms 5.3.1 Convergence of the optimal algorithm}

Figure 3 shows the total transmit power obtained from the iterative algorithm in Algorithm 1 and the low complexity algorithm in Algorithm 2 versus the number of iterations. It is shown that the total transmit power obtained by the iterative algorithm converges to the optimality rapidly in the iteration, and the low complexity algorithm performs close to the iterative algorithm. Moreover, the total transmit power increases with the data rate requirement.

\subsubsection{Comparison with other schemes}

The EE achieved by the proposed iterative algorithm is shown in Figure 4 versus the system's rate, where the system's rate is given as the numerator of the EE defined in (3). Considering that there are no comparable methods available in the literature, we compare with equal bit allocation (EBA) between the desirable and interfering CDIs for each user and equal power allocation (EPA) among multiple users, which are widely applied in prevalent limited feedback systems. To implement the scheme of 'EBA+EPA, we solve optimization problem (6) under two additional constraints: $p_{b_{k}}=p$ and $B_{\ell, b_{k}}=B_{b_{k}} / N_{b}$ 


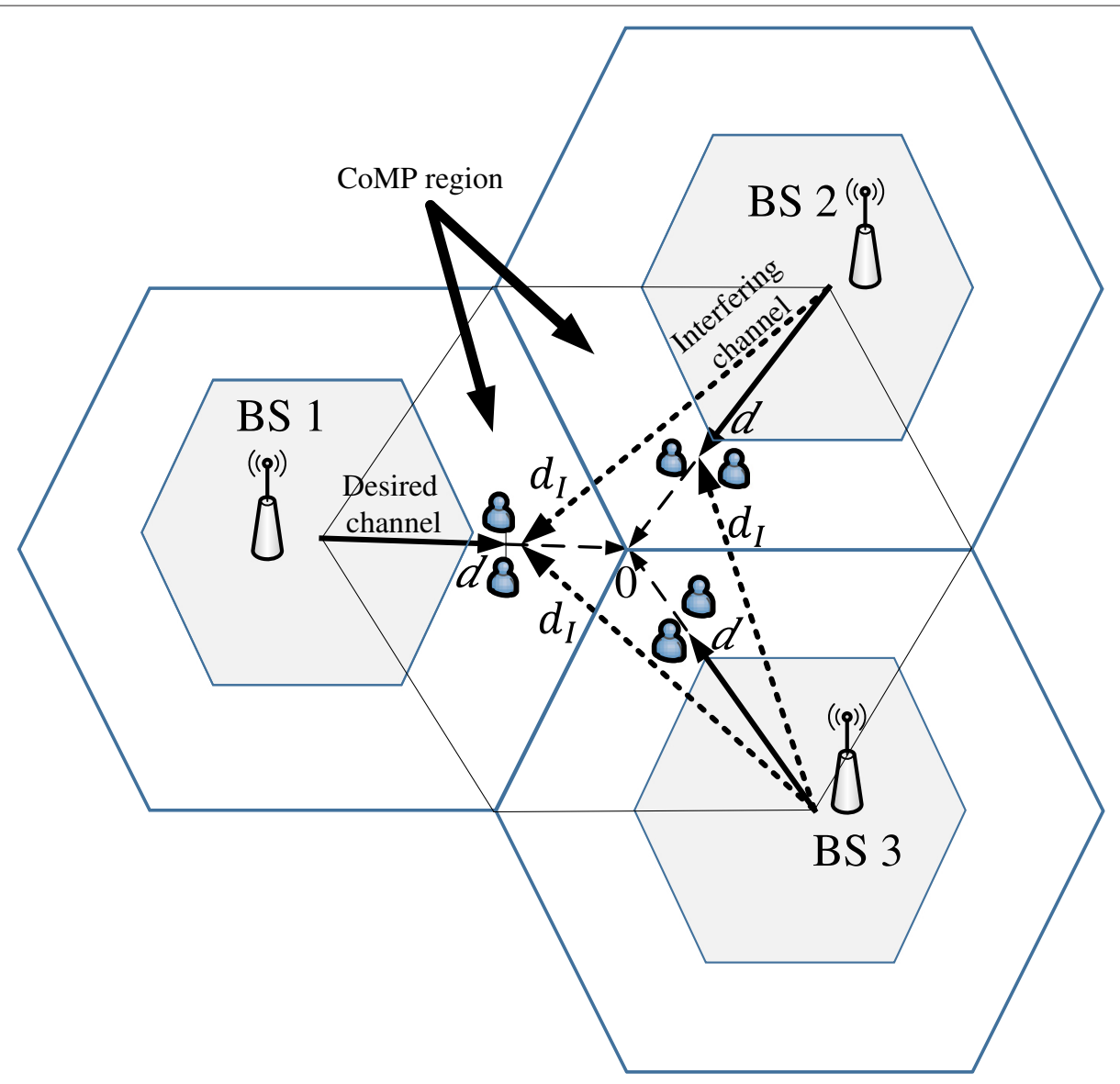

Figure 1 Topology of the simulated CoMP-CB systems.

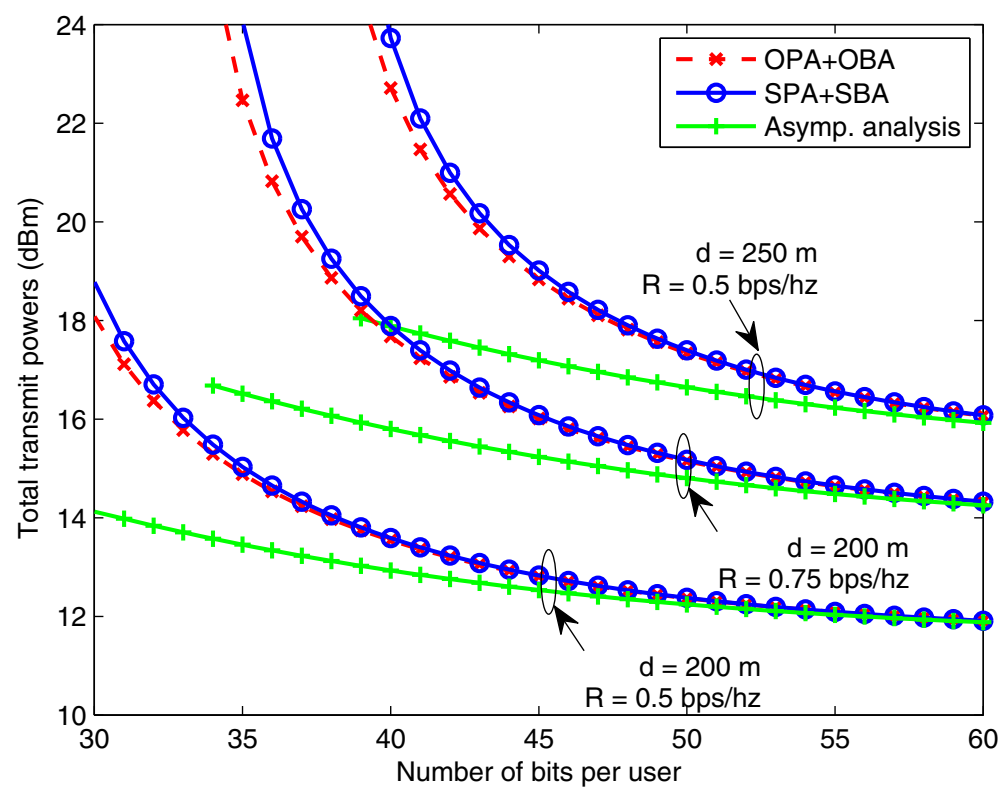

Figure 2 Total transmit power versus the number of bits for the CDI feedback of each user. 


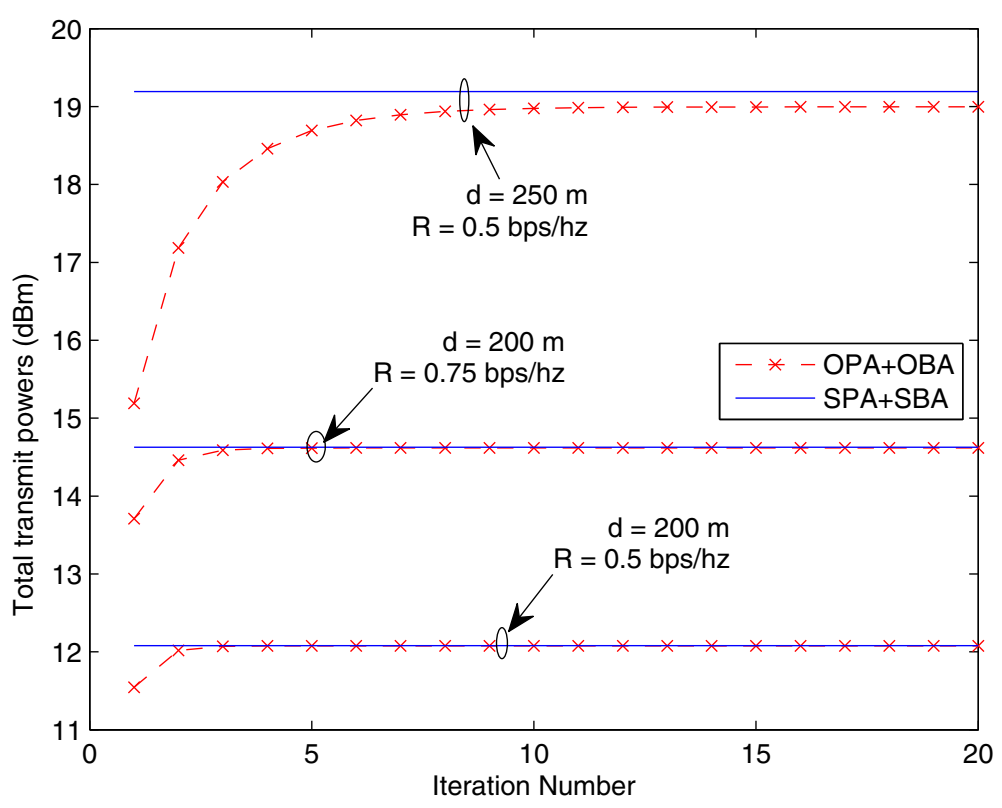

Figure 3 Convergence of the iterative algorithm.

for user $b_{k}(\ell=1,2,3)$. To implement the scheme of 'OPA+EBA,' we fix the equal bit allocation in the algorithm of Algorithm 1, and iterate the transmit powers only. The scheme of 'OPA+EBA' provides the optimal solution for power allocation when the bit allocation is fixed, which can serve as the performance upper bound for any other power allocation algorithms $[21,25]$.
The simulation results show that with equal bit allocation, the optimal power allocation can remarkably improve EE in spite of user locations. Moreover, the EE can be further improved by using the optimal bit allocation. From $d=250$ to $d=200 \mathrm{~m}$, the EE gain of the optimal bit allocation over equal bit allocation increases significantly, since it is more beneficial to use

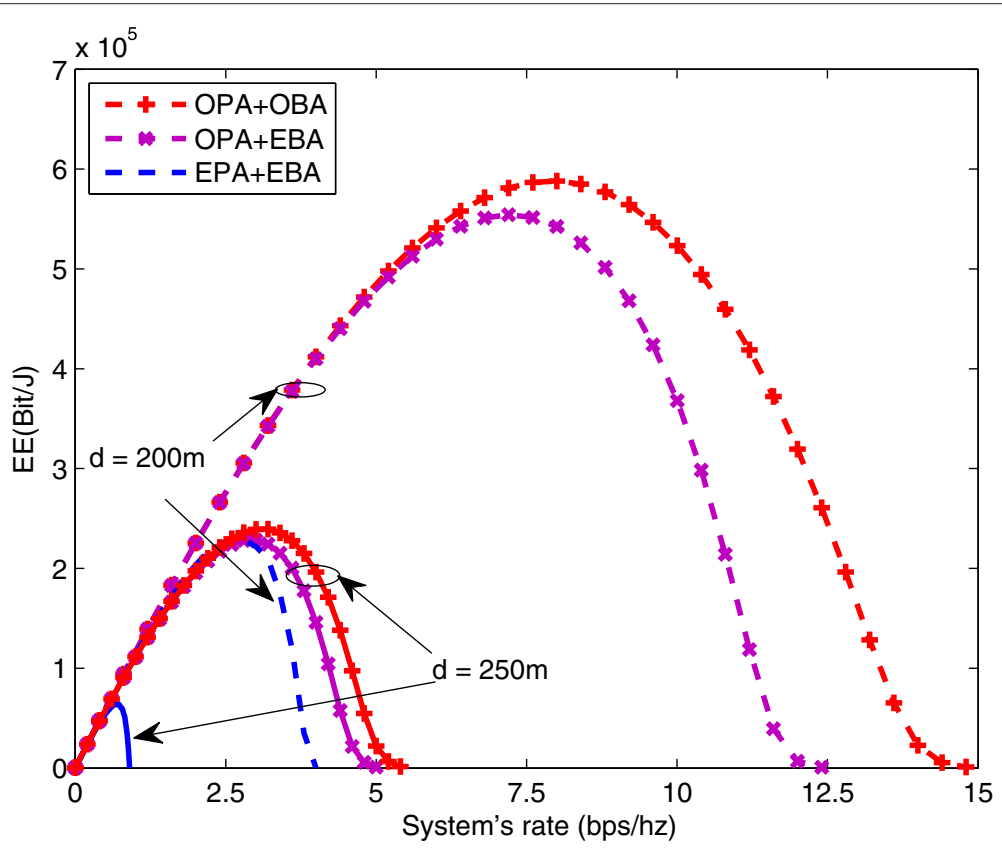

Figure 4 EE under different user locations. $p_{C}=50 \mathrm{~W}[38], \rho_{1}=1 / 2, \rho_{2}=\rho_{3}=\frac{1}{4}$. 


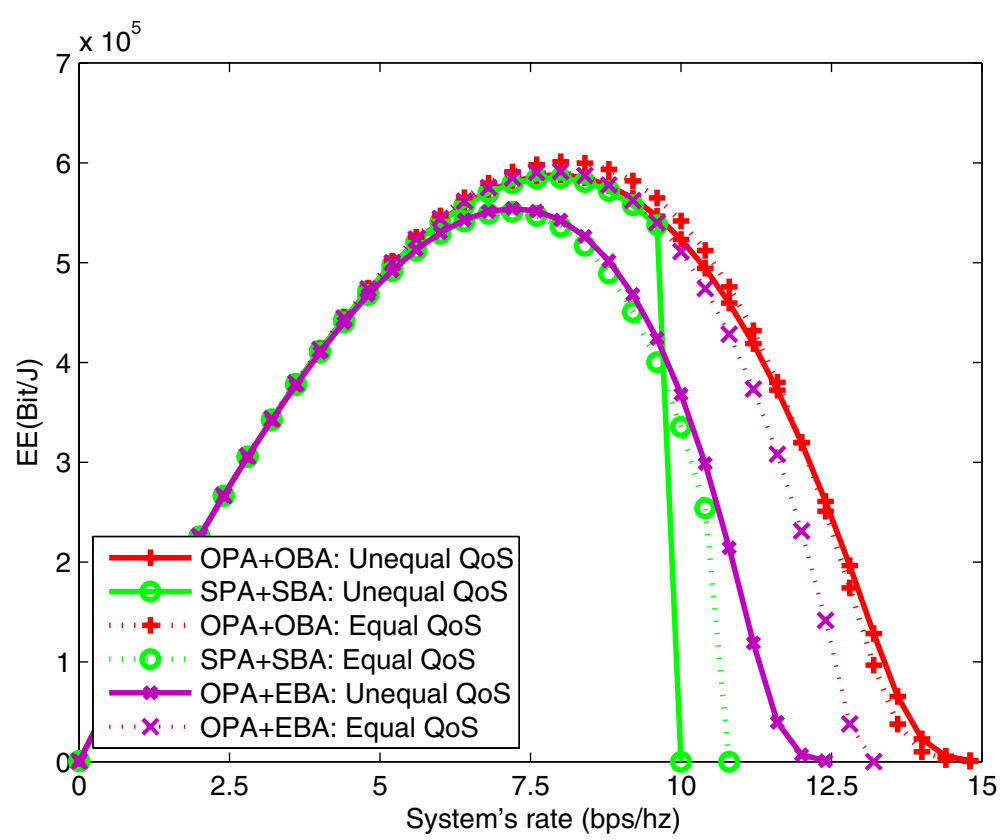

Figure 5 EE of optimal and suboptimal algorithms, $p_{C}=50 \mathrm{~W}, d=200 \mathrm{~m}$. Equal QoS: $\rho_{1}=\rho_{2}=\rho_{3}=1 / 3$; unequal QoS: $\rho_{1}=1 / 2, \rho_{2}=\rho_{3}=\frac{1}{4}$.

the bit allocation for exploiting the heterogeneity of largescale channel gains from desired and interfering BSs. The results highlight the importance of joint power and bit allocation to improve the EE in limited feedback CoMP system.

The EE achieved by the proposed iterative algorithm (OPA+OBA) and low complexity algorithm (SPA+SBA) versus the system's rate is shown in Figure 5, where $p_{c}=$ $50 \mathrm{~W}$ and $d=200 \mathrm{~m}$. We consider two QoS requirements for the users: the equal QoS, where $\rho_{1}=\rho_{2}=\rho_{3}=1 / 3$; the unequal QoS, where $\rho_{1}=1 / 2$ and $\rho_{2}=\rho_{3}=\frac{1}{4}$.

The results show that the EE gap between the iterative and low complexity algorithms is small for low system's rate but large for high system's rate. For the case of high

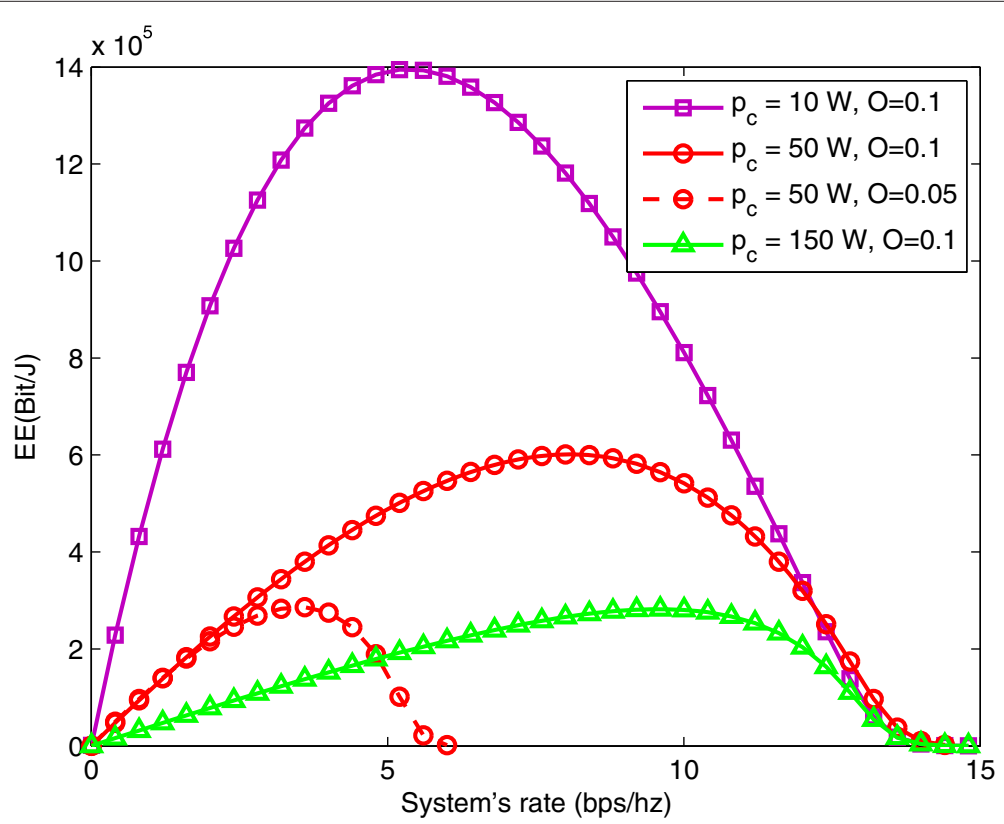

Figure 6 EE under different circuit powers and outage probability constraints. Equal QoS: $\rho_{1}=\rho_{2}=\rho_{3}=\frac{1}{3}$. 


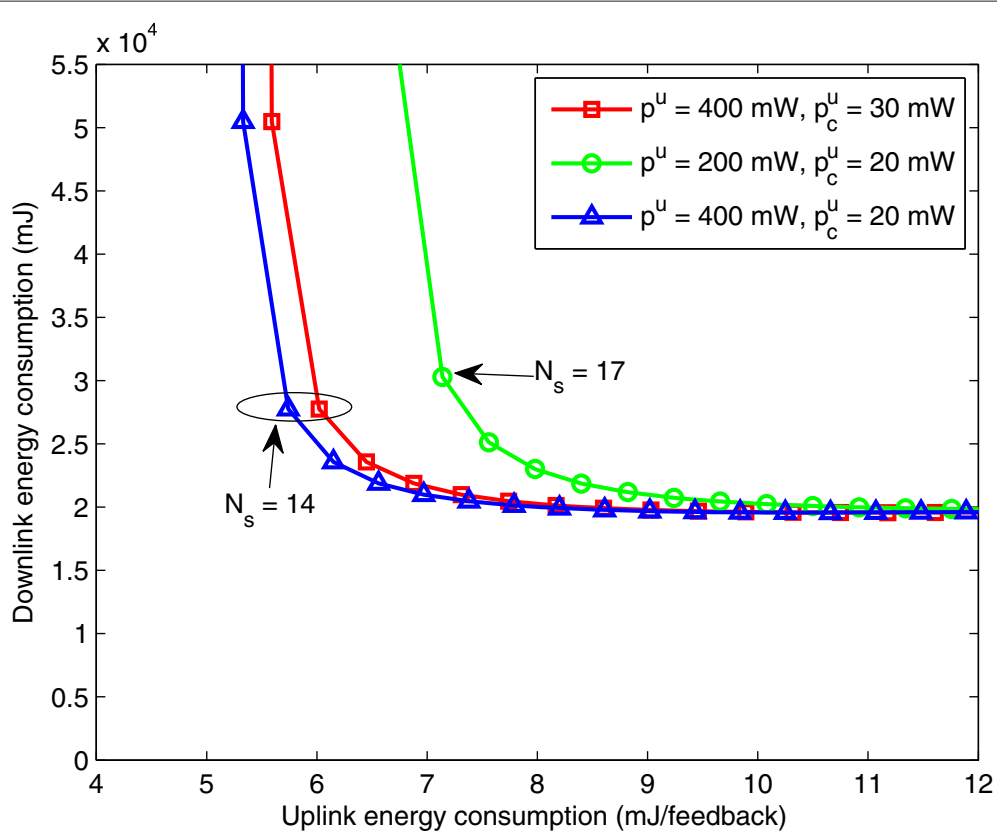

Figure 7 Trade-off between the downlink and uplink energy consumptions. Equal QoS: $\rho_{1}=\rho_{2}=\rho_{3}=1 / 3$.

system's rate under equal QoS, the users with high data rate requirement are with large elements in $\boldsymbol{A}$ as defined in (23). This makes the maximum eigenvalue of $\boldsymbol{A}$ close to 1 and results in the infeasibility for power allocation in (21) as analyzed in Section 4.3. The infeasibility events occur more frequently for the case with unequal QoS, since the users in the first cell with $\rho_{1} R$ are with even larger elements in $\boldsymbol{A}$. As shown in the figure, the performance gain of the optimal bit allocation over the equal bit allocation is larger under unequal QoS requirement than under equal QoS requirement. Moreover, under equal bit allocation, we can find that the optimal bit allocation under unequal QoS is inferior to that under equal QoS. This is because unequal QoS makes the interference among the users more heterogeneous, such that equal bit allocation becomes more improper.

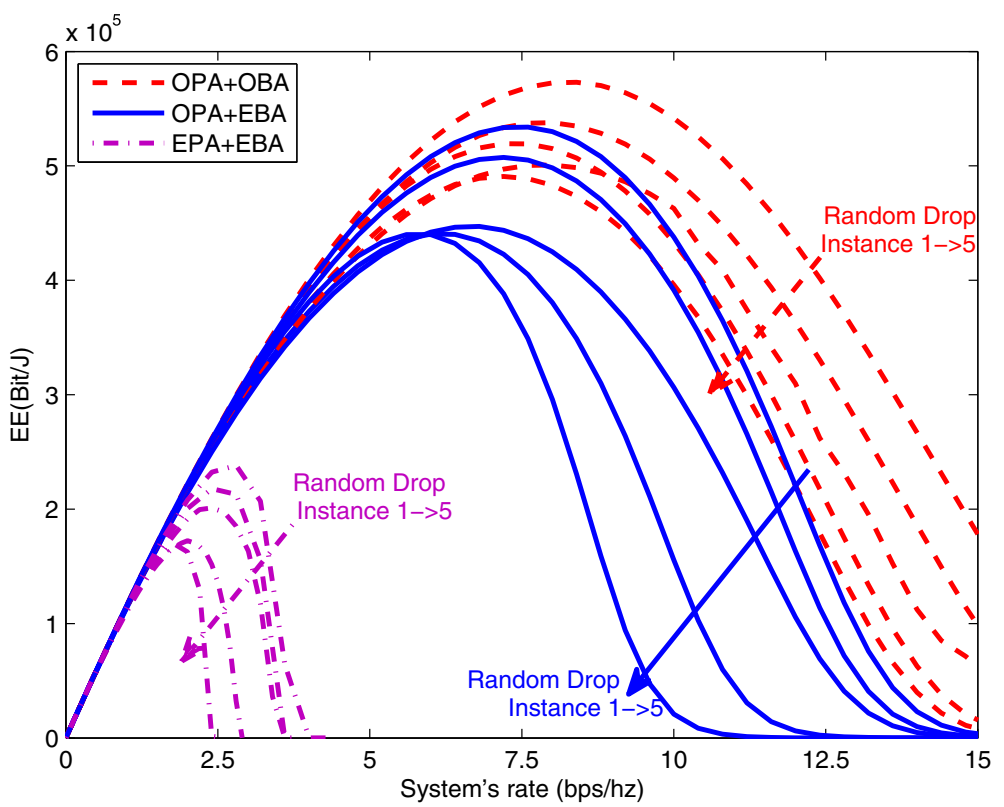

Figure 8 Random drops of users in CoMP-CB system. Equal QoS: $\rho_{1}=\rho_{2}=\rho_{3}=1 / 3$. 


\subsubsection{Impact of circuit power consumption and outage probability}

In Figure 6, we present the EE achieved by the proposed optimal algorithm under different circuit power and outage probability for the case with equal QoS requirement where $d=200 \mathrm{~m}$. In addition to $p_{c}=50 \mathrm{~W}$, two other circuit power consumptions are considered: low circuit power case where $p_{c}=10 \mathrm{~W}$ and high circuit power case where $p_{c}=150 \mathrm{~W}$ [2]. Two target outage probabilities are considered: 0.1 and 0.05 . The simulation results show that the EE decreases with the outage probability. When the outage probability is the same, the EE decreases as the circuit power consumption increases. Since the circuit power consumption does not affect the feasibility of the proposed algorithm, the EE with the same outage probability under different circuit power consumptions drops to zero at the same value of system's rate.

\subsection{Trade-off between downlink and uplink energy consumptions}

In Figure 7, we show the downlink and uplink energy consumptions, where the iterative algorithm in Algorithm 1 is used. We assume that there are $T=100$ slots in downlink transmission per uplink feedback and consider $d=250$ $\mathrm{m}$ and individual data rate requirement of $0.5 \mathrm{bps} / \mathrm{hz}$. Since in the uplink the BSs receive the CDI feedback from the users, we also consider the circuit power consumed at the BS for reception. The downlink circuit power for transmission and reception are respectively $p_{c}=50 \mathrm{~W}$ and $p_{c}=42 \mathrm{~W}$. The energy consumption in uplink is the energy consumed by the circuit power $p_{c}^{u}$ and transmit power $p^{u}$ in the $N_{s}$ feedback slots at each user. The circuit power consumption for users are from [39]. We set the duration of each slot as $1 \mathrm{~ms}$ for both downlink transmission and uplink feedback.

As shown in the figure, for the same values of $p_{c}^{u}$ and $p^{u}$, the more energy are consumed at each user, the less energy are consumed at the BSs. The energy consumption in downlink when $p_{c}^{u}=20 \mathrm{~mW}, p^{u}=200 \mathrm{~mW}$ and $N_{s}=$ 17 is approximately equal to that when $p_{c}^{u}=20 \mathrm{~mW}, p^{u}=$ $400 \mathrm{~mW}$, and $N_{s}=14$. This is because with higher uplink transmit power more bits can be fed back in each time slot and thereby the number of the time slots reduces.

In all three cases, the downlink energy consumption first drops dramatically and then remains constant as the uplink energy consumption increases. This is because when the uplink energy consumption is low, the total number of bits of each user is small such that the MUI and ICI in the system severely degrades the EE performance. The nonlinear impact of the number of bits for feedback on the downlink performance leads to a dramatic change on the downlink energy consumption. However, as the uplink energy consumption increases, the total number of bits of each user becomes large such that the MUI and ICI in the system approaches zero, then the downlink energy consumption becomes unchanged.

In practice, we can judiciously select the number of feedback slots $N_{s}$, i.e., the total number of feedback bits for each user, to trade-off the downlink and uplink energy consumptions.

\subsection{Performance evaluation for general user locations}

We investigate the impact of general user locations on the performance of the proposed algorithm in Figure 8. The second scenario for random user locations in Figure 1 is considered. We consider equal QoS requirement and assume that each user is randomly dropped in the CoMP region with a minimum distance of $200 \mathrm{~m}$ to their serving BSs in the cell-edge.

Figure 8 provides five instances of EE performance under different date rates for CoMP system with randomly distributed users, where the schemes of 'EPA+EBA,' 'OPA+EBA', and 'OPA+OBA' are evaluated. The five instances are selected randomly, and in each instance six users are dropped independently and uniformly in the CoMP region. As shown in Figure 8, similar to Figure 4 where users are symmetrically distributed at fixed locations, it is also of significance to improve the EE performance for limited feedback CoMP-CB system with users at general user locations by using the proposed joint power and bit allocation algorithm.

\section{Conclusions}

We studied energy-efficient power and bit allocation for downlink limited-feedback CoMP-CB systems under the individual date rate requirement and feedback overhead constraint for each user. An optimal solution and a low complexity suboptimal algorithm were respectively proposed to allocate the transmit powers among multiple users and allocate the total number of bits of each user for quantizing the desired and interference channel directions. Simulation results have shown that the proposed algorithms improves the EE of the CoMP-CB systems significantly with respect to the equal power and bit allocation. The target data rate and outage probability of each user, the location of the users as well as circuit power consumption have a large impact on the EE of the system. We found that the energy consumed at the coordinated BSs first reduces rapidly with the increase of energy consumed at each user but remains almost constant when the uplink energy consumption further increases. This suggests that the energy consumption at the BSs can be reduced significantly with a minor increase of the energy consumption at each user if the total number of feedback bits for the user is carefully assigned. 


\section{Competing interests}

The authors declare that they have no competing interests.

\section{Acknowledgements}

This work is supported by the National Natural Science Foundation of China (NSFC) under Grant 61120106002, the National Basic Research Program of China, 973 Program 2012 CB316003 and the National High Technology Research and Development Program of China (No. 2014AA01A703).

\section{Received: 10 July 2014 Accepted: 30 March 2015}

\section{Published online: 01 May 2015}

\section{References}

1. GY Li, Z Xu, C Xiong, C Yang, S Zhang, Y Chen, S Xu, Energy-efficient wireless communications: tutorial, survey, and open issues. IEEE Wireless Commun. Mag. 18(6), 28-35 (2011)

2. ICT-EARTH, Energy Efficiency Analysis of the reference systems, areas of improvements and target breakdown (2011). https://www.ict-earth.eu

3. C Yang, S Han, X Hou, AF Molisch, How to design CoMP to achieve its promised potential?. IEEE, Wireless Commun. Mag. (invited paper) 20(1), 67-74 (2013)

4. DJ Love, RW Heath Jr, Equal gain transmission in multiple-input multiple-output wireless systems. IEEE, Trans. Commun. 51(7), 1102-1110 (2003)

5. R Bhagavatula, RW Heath, Adaptive limited feedback for sum-rate maximizing beamforming in cooperative multicell systems. IEEE, Trans. Signal Process. 59(2) (2011)

6. N Lee, W Shin, Adaptive feedback scheme on K-cell MISO interfering broadcast channel with limited feedback. IEEE Trans. Wireless Commun. 10(2), 401-406 (2011)

7. DJ Love, RW Heath Jr, T Strohmer, in Proc. IEEE Int. Communi. Conf. (ICC). Grassmannian beamforming for multiple-input multiple-output wireless systems (Alaska, US, 2003)

8. N Jindal, MIMO broadcast channels with finite-rate feedback. IEEE, Trans. Inform. Theory. 52(11), 5045-5060 (2006)

9. TYoo, N Jindal, A Goldsmith, Multi-antenna downlink channels with limited feedback and user selection. IEEE J. Select. Areas Commun. 25(7), 1478-1491 (2007)

10. F Yuan, C Yang, G Wang, M Lei, Adaptive channel feedback for coordinated beamforming in heterogeneous networks. IEEE Trans. Wireless Commun. 12(8), 3980-3994 (2013)

11. E Biglieri, J Proakis, S Shamai, Fading channels: information-theoretic and communications aspects. IEEE, Trans. Inform. Theory. 44(6), 1998

12. J Luo, R Yates, P Spasojevic, Service outage based power and rate allocation for parallel fading channels. IEEE Trans. Inform. Theory. 51(7), 2594-2611 (2005)

13. D Wu, R Negi, Effective capacity: a wireless link model for support of quality of service. IEEE Trans. Wireless Commun. 2(4), 630-643 (2003)

14. SV Hanly, DNC Tse, Multiaccess fading channels part II: delay-limited capacities. IEEE Trans. Inform. Theory. 44(7), 2816-2831 (1998)

15. G Caire, G Taricco, E Biglieri, Optimum power control over fading channels. IEEE Trans. Inform. Theory. 45(5), 1468-1489 (1999)

16. C Jiang, L Cimini, in Proc. IEEE Wireless Communi. and Netw. Conf. (WCNC). Energy-efficient transmission for MIMO interference channels (Paris, FR, 2012)

17. MC Gursoy, D Qiao, S Velipasalar, Analysis of energy efficiency in fading channels under QoS constraints. IEEE Trans. Wireless Commun. 8(8), 4252-4263 (2009)

18. A Helmy, L Musavian, T Le-Ngoc, in IEEE Int. Conf. Communi. (ICC) Energy-efficient power allocation for multicarrier systems with delay-outage probability constraints (Budapest, HU, 2013)

19. G Caire, N Jindal, M Kobayashi, N Ravindran, Multiuser MIMO downlink achievable rates with downlink training and channel state feedback. IEEE Trans. Inform. Theory. 56(6), 1478-1491 (2010)

20. Z Xu, C Yang, GY Li, Y Liu, S Xu, Energy-efficient CoMP precoding in heterogeneous networks. IEEE Trans. Signal Process. 62(4), 1005-1017 (2014)

21. M Chiang, P Hande, T Lan, CW Tan, Power Control in Wireless Cellular Networks, vol. 2. (Now publishers, US, 2008)

22. Yates RD, A framework for uplink power control in cellular radio systems. IEEE J. Sel. Areas Commun. 13(7), 1341-1347 (1995)
23. H Dahrouj, W Yu, Coordinated beamforming for the multicell multi-antenna wireless system. IEEE, Trans. Wireless Commun. 9(5), 1748-1759 (2010)

24. S Kandukuri, S Boyd, Optimal power control in interference limited fading wireless channels with outage-probability specifications. IEEE Trans. Wireless Commun. 1(1), 46-55 (2002)

25. J Papandriopoulos, J Evans, S Dey, Optimal power control for Rayleigh-faded multiuser systems with outage constraints. IEEE Trans. Wireless Commun. 4(6), 2705-2715 (2005)

26. CW Tan, in Proc. IEEE INFOCOM. Optimal power control in Rayleigh-fading heterogeneous networks (Shanghai, CN, 2011), pp. 2552-2560

27. D Jaramillo-Ramirez, M Kountouris, E Hardouin, in Proc. IEEE Int. Symp. on Pers., Indoor and Mobile Radio Communi.(PIMRC). Coordinated multi-point transmission with imperfect channel knowledge and other-cell interference (Sydney, AU, 2012)

28. B Khoshnevis, W Yu, Bit allocation laws for multi-antenna channel quantization: multi-user case. IEEE Trans. Signal Process. 59(5), 2270-2283 (2011)

29. S Boyd, L Vandenberghe, Convex Optimization. (Cambridge University Press, New York, NY, US, 2004)

30. Z Xu, C Yang, GY Li, S Zhang, Y Chen, S Xu, Energy-efficient configuration of spatial and frequency resources in MIMO-OFDMA systems. IEEE Trans. Commun. 61(2), 564-575 (2013)

31. Y Zhang, RS Cheng, in Proc. IEEE Wireless Communi. and Netw. Conf. (WCNC). High SNR performance of antenna selection in MISO interference channel with zero-forcing and limited feedback (Paris, FR, 2012)

32. C Li, J Zhang, KB Letaief, in Proc. IEEE Int. Conf. Commun. (ICC). Energy efficiency analysis of small cell networks (Budapest, HU, 2013)

33. JB Rao, AO Fapojuwo, On the tradeoff between spectral efficiency and energy efficiency of homogeneous cellular networks with outage constraint. IEEE Trans. Vehi. Technol. 62(4), 1801-1814 (2013)

34. S Han, C Yang, AF Molisch, Spectrum and energy efficient cooperative base station doze. IEEE J. Select. Areas Commun. 32(2), 285-296 (2013)

35. M Kountouris, JG Andrews, Downlink SDMA with limited feedback in interference-limited wireless networks. IEEE Trans. Wireless Commun. $11(8), 2730-2741$ (2012)

36. Y Huang, CW Tan, BD Rao, in Proc. IEEE Globe Telcommun. Conf.(GLOBECOM). Outage balancing in multiuser MISO networks: network duality and algorithms (California, US

37. J-C Kuang, Applied Inequalities (Chinese), 3rd edn. (Hunan Education Press, Hunan, CN, 1993)

38. G Auer, V Giannini, C Desset, I Godor, P Skillermark, M Olsson, MA Imran, D Sabella, MJ Gonzalez, O Blume, A Fehske, How much energy is needed to run a wireless network?. IEEE Wireless Commun. Mag. 18(5), 40-49 (2011)

39. G Miao, Energy-efficient uplink multi-user MIMO. IEEE, Trans. Wireless Commun. 12(5), 2302-2313 (2013)

\section{Submit your manuscript to a SpringerOpen ${ }^{\circ}$ journal and benefit from:}

- Convenient online submission

- Rigorous peer review

- Immediate publication on acceptance

- Open access: articles freely available online

- High visibility within the field

- Retaining the copyright to your article

Submit your next manuscript at $\mathbf{s p r i n g e r o p e n . c o m ~}$ 